\title{
Axonal Targeting of the Serotonin Transporter in Cultured Rat Dorsal Raphe Neurons Is Specified by SEC24C- Dependent Export from the Endoplasmic Reticulum
}

\author{
Therese R. Montgomery, ${ }^{1,3 *}$ Thomas Steinkellner, ${ }^{1 *}$ Sonja Sucic, ${ }^{1}$ Florian Koban, ${ }^{1}$ Stefan Schüchner, ${ }^{2}$ Egon Ogris, ${ }^{2}$ \\ Harald H. Sitte, ${ }^{1}$ and Michael Freissmuth ${ }^{1}$ \\ ${ }^{1}$ Institute of Pharmacology, Center of Physiology and Pharmacology and 2Department of Molecular Genetics, Medical University of Vienna, A-1090 Vienna, \\ Austria, and ${ }^{3}$ UCD Conway Institute of Biomolecular and Biomedical Research, University College Dublin, Belfield, Dublin 4, Ireland
}

\begin{abstract}
Export of the serotonin transporter (SERT) from the endoplasmic reticulum (ER) is mediated by the SEC24C isoform of the coatomer protein-II complex. SERT must enter the axonal compartment and reach the presynaptic specialization to perform its function, i.e., the inward transport of serotonin. Refilling of vesicles is contingent on the operation of an efficient relay between SERT and the vesicular monoamine transporter-2 (VMAT2). Here, we visualized the distribution of both endogenously expressed SERT and heterologously expressed variants of human SERT in dissociated rat dorsal raphe neurons to examine the role of SEC24C-dependent ER export in axonal targeting of SERT. We conclude that axonal delivery of SERT is contingent on recruitment of SEC24C in the ER. This conclusion is based on the following observations. (1) Both endogenous and heterologously expressed SERT were delivered to the extensive axonal arborizations and accumulated in bouton-like structures. (2) In contrast, SERT- ${ }^{607} \mathrm{RI}^{608}-\mathrm{AA}$, in which the binding site of SEC24C is disrupted, remained confined to the microtubule-associated protein 2-positive somatodendritic compartment. (3) The overexpression of dominant-negative SEC24C-D ${ }^{796} \mathrm{~V} / \mathrm{D}^{797} \mathrm{~N}$ (but not of the corresponding SEC24D mutant) redirected both endogenous SERT and heterologously expressed yellow fluorescent protein-SERT from axons to the somatodendritic region. (4) SERT-K ${ }^{610} \mathrm{Y}$, which harbors a mutation converting it into an SEC24D client, was rerouted from the axonal to the somatodendritic compartment by dominant-negative SEC24D. In contrast, axonal targeting of the VMAT2 was disrupted by neither dominant-negative SEC24C nor dominant-negative SEC24D. This suggests that SERT and VMAT2 reach the presynaptic specialization by independent routes.
\end{abstract}

Key words: axonal delivery; raphe neurons; serotonin transporter; somatodendritic targeting; vesicular monoamine transporter-2

\section{Introduction}

In the adult mammalian brain, the vast majority of serotonergic neuronal somata are restricted to tegmental and pontine raphe nuclei. These neurons produce elaborate axonal projections that innervate virtually all areas of the brain. Serotonin (5-HT) elicits its presynaptic and postsynaptic action by binding to 16 distinct

Received July 15, 2013; revised March 4, 2014; accepted March 31, 2014.

S.SC. and E.O. contributed unpublished reagents/analytic tools; H.H.S. and M.F. designed research; T.R.M., T.S., S.Su., and F.K. performed research; T.R.M., T.S., S.Su., F.K., H.H.S., and M.F. analyzed data; T.R.M. and M.F. wrote the paper.

This work was supported by Austrian Science Fund (FWF)-sponsored Collaborative Research Program SFB-35 and the FWF-funded doctoral programs CCHD (W1205-B09) and MolTag (W1232-B11). T.R.M is a recipient of a Health Research Board/Marie-Curie postdoctoral mobility research fellowship. We thank Cornelius Gross (European Molecular Biology Laboratory, Monterotondo, Italy) for providing SERT-CRE mice, Yelena Kanter, David Sulzer, and Stephan Rayport (Department for Neurology and Psychiatry, Columbia University, New York, NY) for their assistance in and advice on establishing ex vivo neuronal cultures, and Joachim Geyer for the plasmid encoding GFP-VMAT2. We are also grateful to Marion Gröger and Sabine Rauscher of the Core Facility Imaging Unit (Medical University of Vienna, Vienna, Austria) for their assistance in confocal imaging.

*T.R.M. and T.S. contributed equally to this work.

Correspondence should be addressed to Michael Freissmuth, Institute of Pharmacology, Center of Physiology and Pharmacology, Medical University of Vienna, Waehringer Strasse 13a, A-1090 Vienna, Austria. E-mail: michael.freissmuth@meduniwien.ac.at.

T. R. Montgomery's present address: Athlone Institute of Technology, Dublin Road, Athlone, Ireland.

DOI:10.1523/JNEUROSCI.2991-13.2014

Copyright $\odot 2014$ the authors $\quad 0270-6474 / 14 / 346344-08 \$ 15.00 / 0$
G-protein-coupled/metabotropic receptors and to ionotropic 5- $\mathrm{HT}_{3}$ receptors (Kroeze et al., 2012). Invariably, signaling is terminated by removal of 5-HT from the synaptic cleft by the serotonin transporter [SERT; SLC6A4 (solute carrier family 6 [neurotransmitter transporter], member 4)]. SERT functions in a relay system with the vesicular monoamine transporters (in the CNS, predominantly VMAT2/SLC18A2; Kristensen et al., 2011). This allows for efficient retrieval of 5-HT and refilling of the synaptic vesicles. Hence, the action of SERT not only shapes the duration of neurotransmission by clearing 5-HT but also determines the magnitude of the quantal signal by defining the size of the vesicular 5-HT pool. The medical relevance of SERT is obvious because the transporter is the prime target in the treatment of depression: selective serotonin reuptake inhibitors (SSRIs) are the most widely prescribed antidepressants (Kroeze et al., 2012). SERT is also targeted by recreational drugs, i.e., 3,4-methylenedioxy-methamphetamine (MDMA; "ecstasy") and related compounds; their neurotoxic action is also contingent on their SERT-mediated accumulation in target neurons (Steinkellner et al., 2011).

All neurotransmitter transporters are produced in the endoplasmic reticulum (ER) of the neuronal soma; for their eponymous action, they must reach the presynaptic specialization by 
entering the secretory pathway. The machinery that moves cargo proteins through the secretory pathway has been described previously in considerable detail (Anitei and Hoflack, 2012; Gillon et al., 2012; Zanetti et al., 2012). However, it is not so clear where specific sorting decisions are being made, because only a limited number of cargo molecules have been investigated. Neurotransmitter transporters are a specific case in point because closely related representatives have distinct distributions: for instance, Sodium- and chloride-dependent GABA transporter 1 (GAT-1) is delivered to symmetric synapses (Radian et al., 1990; Minelli et al., 1995). In contrast, SERT is deposited in the vicinity of asymmetric synapses, rather than within the synaptic specialization, and is found along the axonal projections at extrasynaptic sites (Zhou et al., 1998; Tao-Cheng and Zhou, 1999; Miner et al., 2000). We showed previously that GAT-1 can only reach the presynaptic specialization if it is exported from the ER by the coatomer protein-II (COPII) machinery. Variants of GAT-1 that fail to recruit SEC24D do eventually reach the cell surface but are not specifically targeted to the axonal compartment (Farhan et al., 2004, 2008; Reiterer et al., 2008). ER export of SERT differs substantially from GAT-1 and its closest relatives dopamine transporter (DAT) and norepinephrine transporter (NET), which all interact with the SEC24D isoform (Farhan et al., 2007; Sucic et al., 2013): in cell lines, both endogenously and heterologously expressed human SERT is an exclusive client of the SEC24C isoform (Sucic et al., 2011). Here, we used primary dissociated rat dorsal raphe neurons to investigate the role of SEC24C in specifying axonal targeting of SERT. Our experiments show that both endogenous SERT and heterologously expressed SERT variants do not enter the axonal compartment if recruitment of SEC24C is precluded.

\section{Materials and Methods}

Reagents. Cell culture material, supplements, and antibiotics were provided by Invitrogen. All other reagents were of analytical grade. The plasmids encoding the yellow fluorescent protein (YFP)-SERT, mCherry-SERT, YFP-NET, hDAT, YFP-SERT- ${ }^{607}{ }^{R I}{ }^{608}-\mathrm{AA}, \mathrm{CFP}-\mathrm{SEC} 24 \mathrm{C}$, CFP-SEC24C- ${ }^{796}$ DV/D D ${ }^{797} \mathrm{~N}$, YFP-SEC24D- ${ }^{733} \mathrm{DV} / \mathrm{D}^{734} \mathrm{~N}$, YFP-SERT $\mathrm{K}^{610} \mathrm{Y}$, and mCherry-SERT $\mathrm{K}^{610} \mathrm{Y}$ mutants were prepared as described previously (Sucic et al., 2011, 2013). The plasmid coding for GFP-VMAT2 was a kind gift from Joachim Geyer (Justus-Liebig University, Giessen, Germany)

$\mathrm{SERT}^{-1-}$ mice. SERT ${ }^{-1-}$ mice were produced by crossing heterozygous SERT-Cre mice. These mice express Cre recombinase under the transcriptional control of the SERT promoter, leading to the loss of SERT expression on the allele in which Cre recombinase is present (Zhuang et al., 2005). The following offspring results: homozygous SERT-Cre mice $\left(S E R T^{-1-}\right)$, heterozygous SERT-Cre $\left(S E R T^{+/-}\right)$, and wild-type $\left(S E R T^{+/+}\right)$mice. Mice were genotyped by PCR using genomic DNA derived from tail biopsies, using the following primers: SertU, $5^{\prime}$ CATCCGCACCACTGACTGACCA-3' (SERT forward primer); SertL, 5'-GGCACTAACCTCCACCATTCTG-3' (SERT reverse primer); and CreL, 5' -GAACGAACCTGGTCGAAATCAG-3' (CRE reverse primer).

Mammalian cell culture and transfections. HEK293 cells were maintained at $37^{\circ} \mathrm{C}$ in a $5 \% \mathrm{CO}_{2}$ humidified atmosphere, in DMEM supplemented with $10 \%$ fetal calf serum and $1 \%$ penicillin/streptomycin. The cells were seeded onto poly-D-lysine-coated $15 \mathrm{~mm}$ glass coverslips and transiently transfected using Turbofect (Fermentas), according to the instructions of the manufacturer. The dorsal raphe nucleus (i.e., the dorsomedial B7 group of neurons under the cerebral aqueduct) was isolated from 1- to 3-d-old rat pups under a dissecting microscope (Yasufuku-Takano et al., 2008). Cells were dissociated and cultured using a modified protocol adapted from Rayport et al. (1992). Briefly, the dorsal raphe tissue was digested in papain for $20 \mathrm{~min}$ at $37^{\circ} \mathrm{C}$ and subsequently triturated to dissociate the cells using increasingly smaller bore pipette tips. The cells were centrifuged for $4 \mathrm{~min}$ at $500 \times g$ and resus- pended in neuronal medium (Neurobasal A, 2\% B27, 1\% heatinactivated calf serum, $0.4 \mathrm{~mm}$ glutamine, and $50 \mu \mathrm{m}$ kynurenic acid). The neurons were then seeded onto a confluent monolayer of rat glial cells grown on $15 \mathrm{~mm}$ glass coverslips. Glial-derived neurotrophic factor (Millipore) was added to each culture $2 \mathrm{~h}$ after seeding to stimulate the sprouting and maturation of neurons. 5-Fluorodeoxyuridine was added to inhibit the proliferation of glia. After $7 \mathrm{~d}$ in vitro, the neurons were transfected using Lipofectamine2000 (Invitrogen).

Preparation of mouse synaptosomes. Adult mice of either sex were killed by cervical dislocation; their brains were removed immediately. The hippocampi were dissected and homogenized in ice-cold $0.32 \mathrm{M}$ sucrose in PBS containing protease inhibitors (Roche Complete). The suspension was centrifuged for $10 \mathrm{~min}$ at $1000 \times \mathrm{g}$. The resulting supernatant was again centrifuged for $15 \mathrm{~min}$ at $12,600 \times g$ to obtain a second pellet (P2), which was resuspended in Krebs-HEPES buffer (KHB; 25 mM HEPES$\mathrm{NaOH}, \mathrm{pH}$ 7.4, $120 \mathrm{~mm} \mathrm{NaCl}, 5 \mathrm{~mm} \mathrm{KCl}, 1.2 \mathrm{~mm} \mathrm{CaCl}_{2}$, and $1.2 \mathrm{~mm}$ $\mathrm{MgSO}_{4}$ ) supplemented with $5 \mathrm{~mm}$ D-glucose. Protein concentration was determined using the BCA protein assay (Thermo Fisher Scientific).

Preparation of cell lysates. HEK293 cells were transfected using Turbofect (Fermentas) according to the instructions of the manufacturer. Forty-eight hours after transfection, cells were washed three times with ice-cold PBS and lysed in buffer (10 mm Tris-HCl, pH 7.4, $150 \mathrm{~mm} \mathrm{NaCl}$, 1 mM EDTA, $1 \%$ Triton X-100, and 0.1\% SDS) supplemented with protease inhibitors (Roche Complete) and incubated on a rotator for $30 \mathrm{~min}$ at $4^{\circ} \mathrm{C}$. Subsequently, the lysates were centrifuged for $10 \mathrm{~min}$ at $13,000 \times$ $g$. The pellet was discarded, and protein concentration of the supernatant was measured using the BCA protein assay (Thermo Fisher Scientific).

SDS-PAGE and immunoblotting. Synaptosomal membranes or cell lysates $(10-30 \mu \mathrm{g})$ were mixed with an equal volume of $2 \times$ sample loading buffer (125 mM Tris-HCl, pH 6.8, 4\% SDS, 20\% glycerol, $10 \%$ 2-mercaptoethanol, and $0.02 \%$ bromphenol blue), denatured for $30 \mathrm{~min}$ at room temperature, and loaded onto a $10 \%$ SDS polyacrylamide gel. The resolved proteins were electrophoretically transferred onto Protran nitrocellulose membranes (Whatman), which were blocked by an incubation in Tris-buffered saline containing $0.1 \%$ Tween 20 and $5 \%$ bovine serum albumin for $1 \mathrm{~h}$ at room temperature. Incubation with primary antibodies was performed overnight at $4^{\circ} \mathrm{C}$, using the following antibodies: mouse monoclonal anti-SERT H-45, goat polyclonal anti-SERT (sc1458; Santa Cruz Biotechnology), and rabbit polyclonal anti-GFP (Abcam). The immunoreactive bands were visualized by enhanced chemiluminescence (Thermo Fisher Scientific) with horseradish peroxidase-conjugated secondary antibodies.

Immunocytochemistry and confocal microscopy. Cells were fixed using either 3.7\% paraformaldehyde (PFA) for $20 \mathrm{~min}$ at room temperature or a solution containing acetone/methanol (1:1) for $2 \mathrm{~min}$ at room temperature. The cells were washed three times with PBS and incubated for $1 \mathrm{~h}$ at room temperature in blocking/permeabilization buffer $(0.2 \%$ saponin and 5\% goat serum in PBS). The cells were then incubated overnight at $4^{\circ} \mathrm{C}$ with one or more of the following primary antibodies in blocking buffer: mouse monoclonal anti-SERT (H-45); rat monoclonal anti-DAT antibody (MAB369; Millipore Bioscience Research Reagents); goat polyclonal anti-SERT (sc-1458; Santa Cruz Biotechnology); rabbit polyclonal anti-microtubule-associated protein 2 (MAP-2) antibody (AB5622; Millipore Bioscience Research Reagents); rabbit polyclonal anti-tyrosine hydroxylase antibody (AB152; Millipore Bioscience Research Reagents); mouse anti-tryptophan hydroxylase 2 (TPH2) antibody (MAB1488; Acris Laboratories); or rabbit polyclonal anti-VMAT2 antibody (AB1598P; Merck Millipore). The next day, the cells were washed three times in PBS and incubated for $1 \mathrm{~h}$ at room temperature with Alexa Fluor-conjugated secondary antibodies (Invitrogen). Cells were then washed three times in PBS before imaging. Confocal microscopy was performed under oil immersion $(40 \times$ and $63 \times$ objectives, numerical aperture 1.4$)$ using either a Zeiss LSM510 confocal microscope a Zeiss LSM780 confocal microscope equipped with a 32-channel gallium arsenide phosphide detector array. Captured images were analyzed using Zeiss Zen Lite 2012. Single neurons (i.e., solitary cells on a glial island) that (co)expressed constructs (i.e., wild-type or mutated versions of tagged SERT; tagged versions of SEC24C or SEC24D, tagged VMAT2) were selected to assess the trafficking of SERT and VMAT2. Images were printed, and the distribution of 
SERT and/or VMAT2 in the MAP-2-positive and MAP-2-negative compartment was scored by an observer who was blinded to the condition of the experiment (i.e., mutant or wild-type SERT; wild-type or dominantnegative SEC24C or SEC24D).

\section{Results}

Characterization of a monoclonal antibody directed against SERT (H-45)

A monoclonal antibody was raised by immunizing mice against a C-terminal hSERT-GST fusion protein, and the hybridoma supernatants were screened for antibodies that recognized the immunogen with high affinity. This selection led to the identification of clone $\mathrm{H}-45$, which produced an IgG2b/к. We used mice rendered deficient in SERT to verify that this antibody specifically recognized SERT. These synaptosomal preparations were electrophoretically resolved and probed with $\mathrm{H}-45$ : in membranes obtained from wild-type animals, the antibody recognized a diffuse band in the range of $75 \mathrm{kDa}$ and two additional bands that migrated with slightly higher mobility (Fig. $1 A$, top left blot). These bands are likely to represent differentially glycosylated forms (El-Kasaby et al., 2010). They were also recognized by the commercially available (goat) polyclonal antibody SC1458 directed against SERT (Fig. 1A, bottom left blot), but they were absent in SERT-deficient animals (Fig. 1A, lanes labeled SERT$\mathrm{KO})$. SERT is closely related to NET and DAT; because of the small size of the rodent brainstem, serotonergic neurons are in close vicinity to other monoaminergic neurons, in particular the noradrenergic neurons. Accordingly, we also examined any potential cross-reactivity of the antibody with the other monoamine transporters by electrophoretically resolving HEK293 cell membranes expressing tagged versions of SERT, NET, and DAT. The H-45 SERT antibody recognized neither NET nor DAT (data not shown). Finally, we confirmed that the H-45 SERT antibody was suitable for detecting SERT by immunofluorescence: YFP-tagged SERT was visualized in fixed, permeabilized HEK293 cells by indirect immunofluorescence with H-45 (Fig. 1B, top, left image) and by exploiting the YFP tag. Images were captured by confocal microscopy (Fig. 1B, top). As an additional control, HEK293 cells expressing either human DAT (DAT) or YFP-tagged NET (YhN) were fixed and stained with the SERT H-45 antibody (Fig. $1 B$, middle and bottom panels, respectively). The SERT H-45 antibody did neither recognize DAT (which was visualized by the rat monoclonal anti-DAT specific antibody MAB369 from Millipore Bioscience Research Reagents) or YFP-tagged NET when examined by confocal microscopy.

\section{Characterization of rat dorsal raphe neurons}

The dorsomedial portions of the dorsal raphe nucleus were dissected from the midbrain of newborn rat pups (P0-P3), and the dissociated B7 group of serotoninergic neurons were maintained in culture. Their serotonergic nature was confirmed by a combination of immunofluorescence imaging (Fig. 2) and functional analyses (data not shown). TPH is the rate-limiting enzyme in 5-HT synthesis. TPH-2 is an isoenzyme of TPH, primarily expressed in the serotonergic neurons of the raphe nuclei (Walther and Bader, 2003). Figure $2 A$ demonstrates the colocalization of SERT (detected with the sc-1458 antibody) and TPH-2 in a rat dorsal raphe neuron. Both the H-45 SERT antibody and the antibody directed against TPH-2 are of murine origin. Hence, it was not possible to stain neurons with these two antibodies simultaneously. However, immunoreactivity detected with the mouse monoclonal SERT H-45 antibody and with the (goat) SERT SC1458 antibody colocalized (data not shown). The mouse SERT
A synaptosomes HEK293 cells

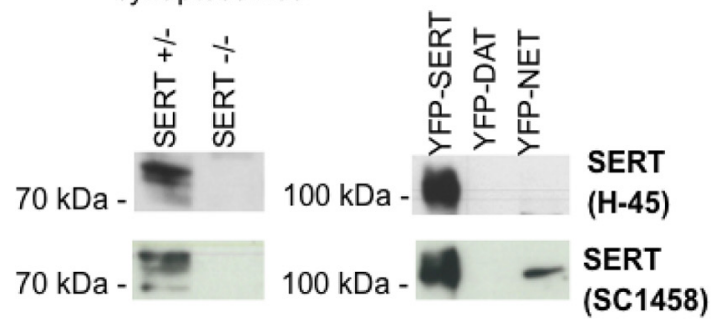

\section{B}

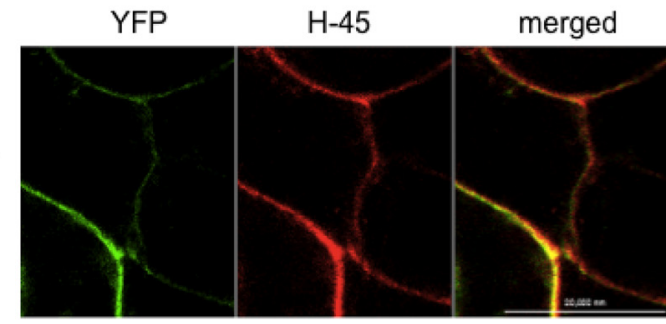

YFP-SERT
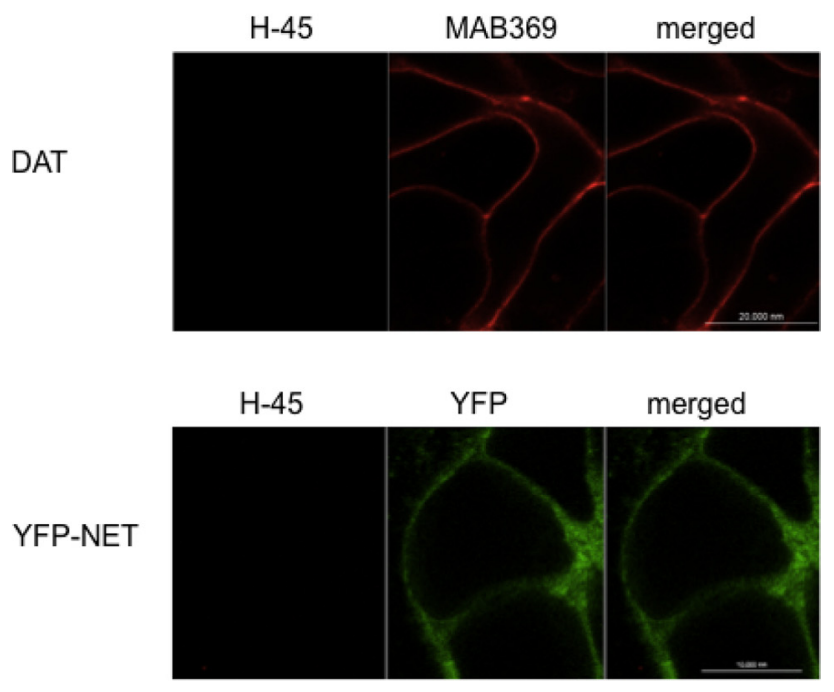

Figure 1. Specificity of the mouse monoclonal (H-45) SERT antibody. A, Hippocampal synaptosomal proteins (15 $\mu \mathrm{g} / \mathrm{lane}$ ) were prepared from wild-type and SERT-deficient mice (left blots); cell membranes (15 $\mu \mathrm{g} / \mathrm{lane}$ ) were prepared from HEK293 cells stably expressing YFPtagged human SERT (YhS), DAT (YhD), and NET (YhN) (right blots). After electrophoretic resolution and transfer of the proteins, immunoreactive bands were detected with the H-45 monoclonal anti-SERT antibody (top row) or with a commercially available SERT antibody (sc1458) (bottom row). B, YFP-tagged SERT expressed in HEK293 cells was visualized by confocal microscopy by either its fluorescent tag or immunostaining of fixed cells with the anti-SERT H-45 antibody using an Alexa Fluor 568-conjugated secondary antibody (top). The cells were washed, and the fluorescence was visualized by confocal microscopy. Merged images are shown on the right side to illustrate the colocalization. HEK293 cells expressing human DAT (DAT, top) or YFP-tagged human NET (YFP-NET, bottom) were fixed and stained with the DAT-directed antibody MAB369 (top) and the anti-SERT antibody H-45. Immunofluorescence and YFP fluorescence were imaged by confocal microscopy. Representative data are shown that were reproduced in at least one independent experiment.

$\mathrm{H}-45$ antibody was more sensitive because it visualized the presence of SERT on thin, multi-branching neuronal extensions that were not identified by the sc-1458 antibody. Given its greater specificity (see above and Fig. 1) and sensitivity, the H-45 antibody was used in all subsequent stainings of endogenous SERT. The immunoreactivity detected by the $\mathrm{H}-45$ antibody resided in an axonal compartment, because it did not colocalize with immunostaining for MAP-2 (Fig. 2B). MAP-2 is a neuron-specific cytoskeletal protein believed to play a role in determining and 


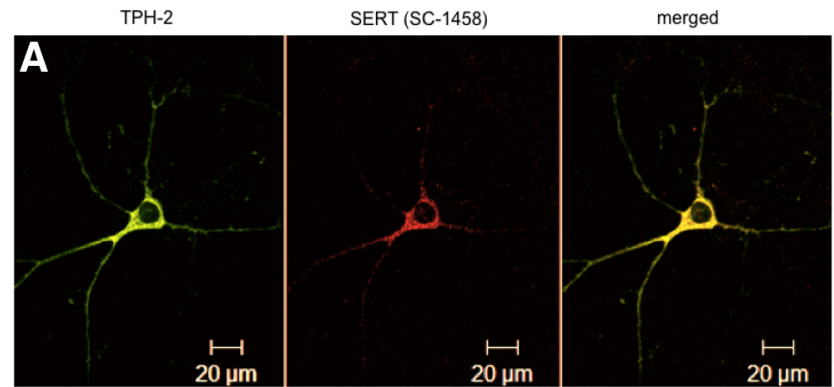

z-stack: merged images for SERT (H-45, green) \& MAP2 (red)
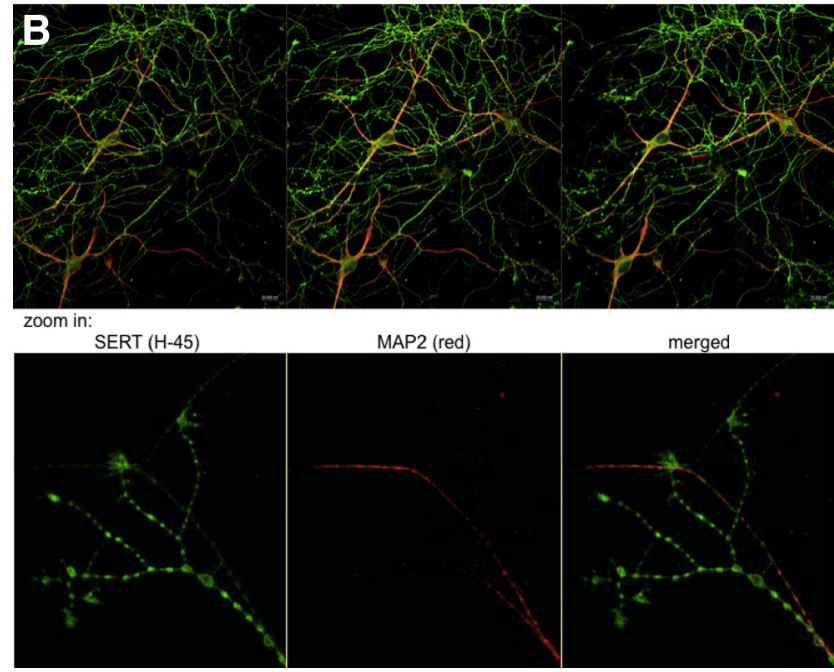

Figure 2. Immunostaining of rat dorsal raphe neurons for SERT and TPH2. Rat dorsal raphe neurons were prepared and cultured as described in Materials and Methods, fixed, and stained with primary antibodies directed against TPH2 (A), SERT (sc-1458 in $\boldsymbol{A} ; \mathrm{H}-45$ in $\boldsymbol{B}$ ), and MAP-2 (B). Immunoreactive material was visualized with Alexa Fluor 488- and Alexa Fluor 568conjugated secondary antibodies. Images were captured by confocal microscopy. The bottom row in $\boldsymbol{B}$ zooms in on the distal end of an axonal arborization to visualize the accumulation of SERT in bouton-like structures and at the tips of the neurite extensions. Data are representative of $\geq 20$ neurons from $\geq 3$ independent preparations. Scale bars, $20 \mu \mathrm{m}$.

stabilizing dendritic shape during neuron development. MAP-2 is used as a standard dendritic marker in morphological studies of dissociated postnatal neurons (Eriksen et al., 2009). These MAP2-negative axonal arborizations contained multiple fluorescent boutons; SERT immunoreactivity was also enriched at the very tips, indicating that SERT was delivered to presynaptic specializations (Fig. 2C, bottom). Finally, it was not surprising that the neuronal soma contained immunoreactivity for SERT (Fig. $2 A, B)$, because SERT is synthesized in the ER of the soma. We also visualized VMAT2 as an axonal marker: immunoreactivity for VMAT2 was also found predominantly in the MAP-2negative compartment (Fig. 3A).

\section{Restricting exogenously expressed YFP-tagged SERT to the somatodendritic compartment}

If a plasmid coding for an mCherry- or a YFP-tagged version of SERT was introduced into raphe neurons, the resulting fluorescent SERT protein was visualized in both the neuronal soma and the thin, multi-branching neuronal extensions (Figs. 3B, 4A). These branches had multiple fluorescent boutons that were adjacent to coexpressed VMAT2 (Fig. 3B), but they did not contain any immunoreactivity for MAP-2, indicating that they represented axonal arborizations (Figs. 3B, 4A). Therefore, we concluded that heterologously expressed SERT was targeted to the same compartments as the endogenous protein visualized by im-
A

VMAT2

MAP-2

merged

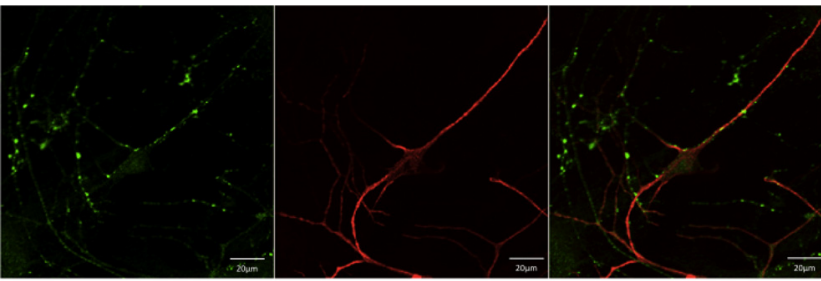

B

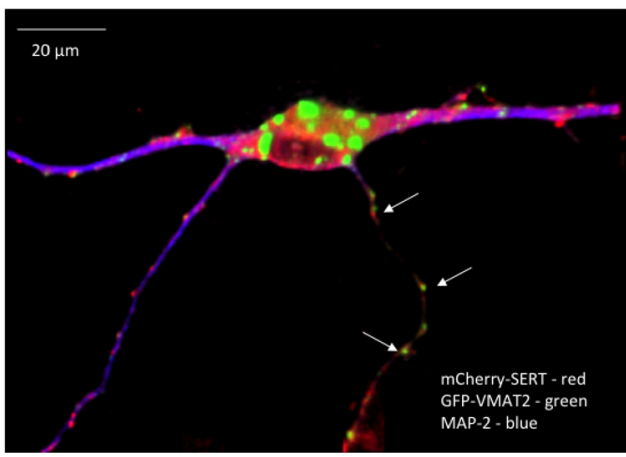

Figure 3. Distribution of endogenous $(\boldsymbol{A})$ and heterologously expressed $(\boldsymbol{B})$ VMAT2. $\boldsymbol{A}$, Rat dorsal raphe neurons were prepared and cultured as described in Materials and Methods, fixed, and stained with primary antibodies directed against VMAT2 and MAP-2. Immunoreactive material was visualized with Alexa Fluor 488- and Alexa Fluor 568-conjugated secondary antibodies. Images were captured by confocal microscopy. A merged image is shown on the right to illustrate the fact that VMAT2 is found predominantly in the MAP-2-negative compartment. $\boldsymbol{B}$, Rat dorsal raphe neurons were cotransfected with plasmids encoding GFP-tagged VMAT2 (green) and mCherry-tagged SERT (red) and stained for MAP-2 by using an Alexa Fluor 405conjugated secondary antibody (blue). A 3D reconstruction was generated from z stacks (slice thickness, $0.5 \mu \mathrm{m}$ ) using NIH Image J (version 1.44p) to highlight the presence of both GFPtagged VMAT2 and mCherry-tagged SERT in the MAP-2-negative compartment in close proximity to each other (white arrows). Scale bars, $20 \mu \mathrm{m}$.

munofluorescence (Figs. 2B, 4A). SLC6 family members contain a conserved RI/RL motif that recruits the SEC24 moiety of the COPII coat; its mutation leads to COPII-independent export (Farhan et al., 2007, Sucic et al., 2011, 2013). Accordingly, we expressed YFP-tagged SERT- ${ }^{607} \mathrm{RI}^{608}-\mathrm{AA}$ in raphe neurons. This mutated transporter was confined to the MAP-2-positive compartment (Fig. 3B). As an alternative approach, we used fluorescently tagged dominant-negative versions of SEC24C $\left(\mathrm{SEC} 24 \mathrm{C}-\mathrm{D}^{796} \mathrm{~V} / \mathrm{D}^{797} \mathrm{~N}\right)$ and SEC24D (SEC24D-0D ${ }^{733} \mathrm{~V} /$ $\left.\mathrm{D}^{734} \mathrm{~N}\right)$ that disrupt the COPII-dependent ER export of SERT (Sucic et al., 2011, 2013) and of GAT-1 (Farhan et al., 2007) and NET (Sucic et al., 2013), respectively. It is evident from Figure $5 B$ that coexpression of a CFP-tagged version of SEC24C-D ${ }^{796} \mathrm{~V} /$ $\mathrm{D}^{797} \mathrm{~N}$ resulted in extensive colocalization of YFP-tagged SERT with MAP-2 and greatly reduced the appearance YFP-SERT in axonal arborizations (Fig. 5B, with pertinent control transfection shown in $A$ ). In contrast, coexpression of YFP-tagged dominantnegative SEC24D-D ${ }^{733} \mathrm{~V} / \mathrm{D}^{734} \mathrm{~N}$ did not affect the ability of mCherry-tagged SERT to enter into the axonal compartment (Fig. 5C).

\section{Preventing entry of endogenous SERT into the axonal compartment by dominant-negative SEC24C}

The observations summarized in Figures 4 and 5 suggested that recruitment of SEC24C was required to direct exogenously expressed SERT into the axonal compartment. We verified that this 
A

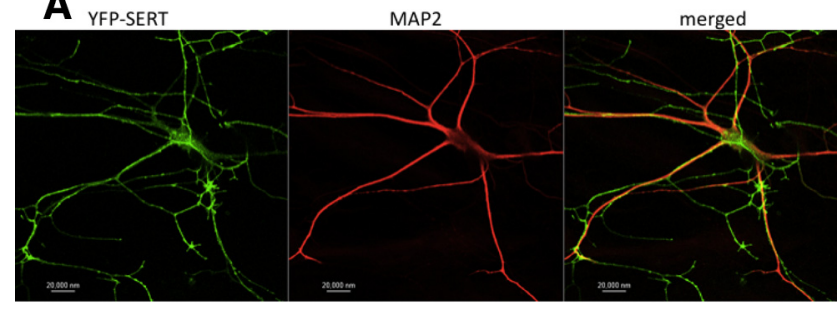

B

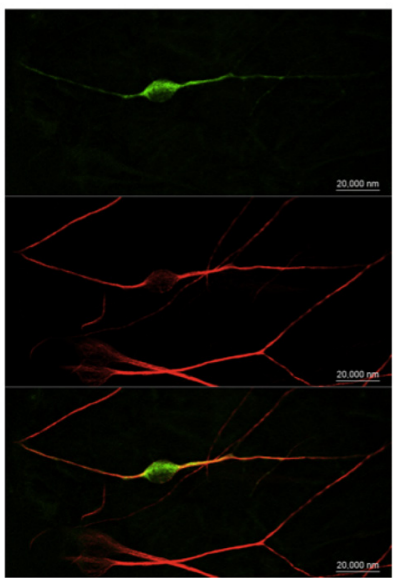

Figure 4. YFP-SERT $-{ }^{607} \mathrm{RI}^{608}-\mathrm{AA}$ fails to reach axonal compartments. Rat dorsal raphe neurons were transfected with plasmids encoding YFP-SERT $(\boldsymbol{A})$ or YFP-SERT- ${ }^{607} \mathrm{RI}^{608}-\mathrm{AA}$ (B) using Lipofectamine2000. After $48 \mathrm{~h}$, the neurons were fixed and stained for MAP-2. MAP-2 staining was detected using an Alexa Fluor 568-conjugated secondary antibody. Images were captured by confocal microscopy. YFP-tagged SERT was found in the MAP-2-negative compartment in 24 of 24 examined neurons; YFP-tagged SERT $-{ }^{607} \mathrm{RI}^{608}$-AA was confined to the MAP2-positive compartment in 20 of 21 examined neurons. Data are from three independent experiments (i.e., 4 individual preparations of rat dorsal raphe neurons that were independently transfected in parallel with a plasmid encoding either YFP-SERT or YFP-SERT$\left.{ }^{607} \mathrm{RI}^{608}-\mathrm{AA}\right)$. Scale bars, $20 \mu \mathrm{m}$.

was also the case for endogenous SERT by resorting again to the use of fluorescently tagged, dominant-negative versions of SEC24. This approach is superior to using siRNAs because the fluorescent tag allows for unambiguous identification of transfected neurons. If neurons were transfected with CFP-tagged wild-type SEC24C, there was not any appreciable effect on the distribution of endogenous SERT: SERT immunoreactivity detected with the $\mathrm{H}-45$ antibody was copiously distributed over the MAP-2-negative bouton-dotted arborizations (Fig. 6A). In contrast, in neurons that expressed dominant-negative CFP-tagged SEC24C-D ${ }^{796} \mathrm{~V} / \mathrm{D}^{797} \mathrm{~N}$, SERT immunoreactivity was confined to the MAP-2-positive compartment (Fig. 6B). Dominantnegative SEC24D was used as an additional control: expression of YFP-tagged SEC24D-D ${ }^{733} \mathrm{~V} / \mathrm{D}^{734} \mathrm{~N}$ was readily visualized in serotonergic neurons, but it did not affect the ability of SERT to enter the MAP-2-negative axonal compartment (Fig. 6C).

\section{Axonal targeting of YFP-tagged SERT-K ${ }^{610} \mathrm{Y}$, an SEC24D client}

Dominant-negative SEC24C may impinge on the formation of bouton-dotted axonal arborizations, an effect that would confound the interpretation. We addressed this question by expressing the mutated version of SERT- $\mathrm{K}^{610} \mathrm{Y}$, which recruits SEC24D rather than SEC24C (Sucic et al., 2013): SERT-K ${ }^{610} Y$ per se reached the MAP-2-negative neurite extensions and accumu-
A

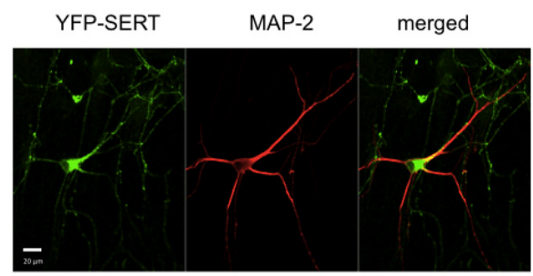

B

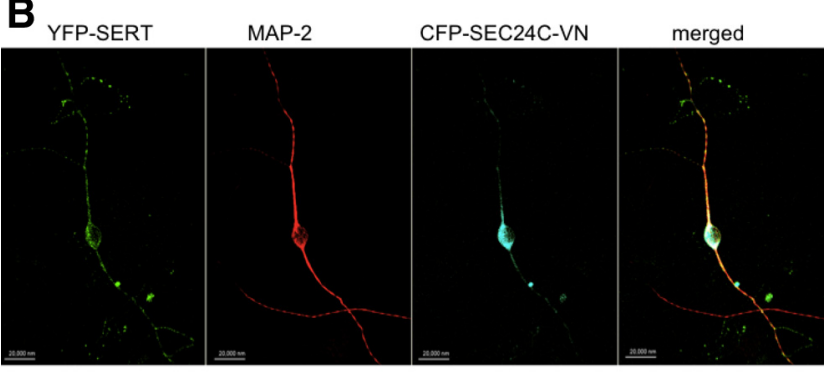

C

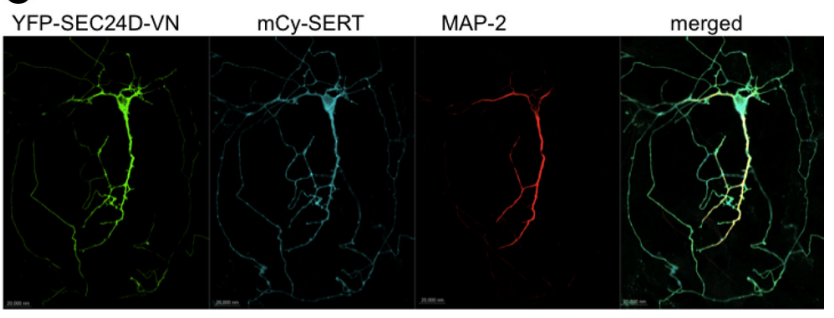

Figure 5. Cotransfection of dominant-negative SEC24C (SEC24C-VN), but not of SEC24D (SEC24D-VN), reduces axonal targeting of YFP-SERT. Rat dorsal raphe neurons were transfected with plasmids encoding YFP-tagged SERT alone $(\boldsymbol{A})$, YFP-tagged SERT and CFPSEC24C-VN (ratio of 1:5; $\boldsymbol{B}$ ), or mCherry-tagged SERT (mCy-SERT) and YFP-SEC24D-VN (ratio of $1: 5 ; C$ ) using Lipofectamine2000. After $48 \mathrm{~h}$, the neurons were fixed and stained for MAP-2. MAP-2 staining was detected using Alexa Fluor 568- or Alexa Fluor 633-conjugated secondary antibodies. Images were captured by confocal microscopy. During coexpression of CFPSEC24C-VN, YFP-tagged SERT was confined to the MAP-2-positive compartment in 18 of 21 examined neurons; in the presence of dominant-negative SEC24D, mCherry-tagged SERT reached the MAP-2-negative compartment in 16 of 18 examined neurons. Data are from four independent experiments (i.e., 4 individual preparations of rat dorsal raphe neurons that were independently transfected in parallel with a plasmid coding for a tagged version of SERT and plasmids encoding (FP-SEC24C-VN or YFP-SEC24D-VN). Scale bars, $20 \mu \mathrm{m}$.

lated in fluorescent boutons in a manner indistinguishable from YFP-tagged wild-type SERT (Figs. 4A, 7A).

Consistent with the switch in SEC24 specificity, coexpression of dominant-negative SEC24D- $\mathrm{D}^{733} \mathrm{~V} / \mathrm{D}^{734} \mathrm{~N}$ resulted in the confinement of fluorescent SERT- $\mathrm{K}^{610} \mathrm{Y}$ to the MAP-2-positive compartment (Fig. 7B). Conversely, in neurons that expressed CFP-tagged SEC24C-D ${ }^{796} \mathrm{~V} / \mathrm{D}^{797} \mathrm{~N}$, YFP-tagged SERT-K ${ }^{610} \mathrm{Y}$ readily reached the arborized, axonal extensions (Fig. $7 C$ ). Finally, we examined the effect of dominant-negative versions of SEC24C and SEC24D on the distribution of VMAT2 (Fig. 8): we visualized the effect of SEC24C-D ${ }^{796} \mathrm{~V} / \mathrm{D}^{797} \mathrm{~N}$ and SEC24C$\mathrm{D}^{796} \mathrm{~V} / \mathrm{D}^{797} \mathrm{~N}$ on the distribution of both heterologously expressed and endogenous VMAT2. Figure 8 shows representative images of the effect of SEC24C-D ${ }^{796} \mathrm{~V} / \mathrm{D}^{797} \mathrm{~N}$ on GFP-tagged VMAT2 (Fig. 8A) and SEC24C-D ${ }^{796} \mathrm{~V} / \mathrm{D}^{797} \mathrm{~N}$ on endogenous VMAT2 (Fig. $8 B$ ). It is evident that neither dominant-negative SEC24C nor dominant-negative SEC24D abolished delivery of VMAT2 to the MAP-2-negative compartment. Similar observations were made with the other combinations, i.e., dominantnegative SEC24C and SEC24D did not preclude axonal delivery of endogenous and heterologously expressed VMAT2, respectively (data not shown). 


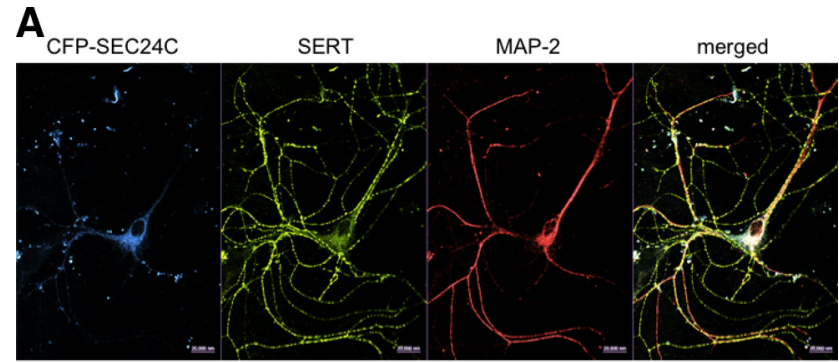

B

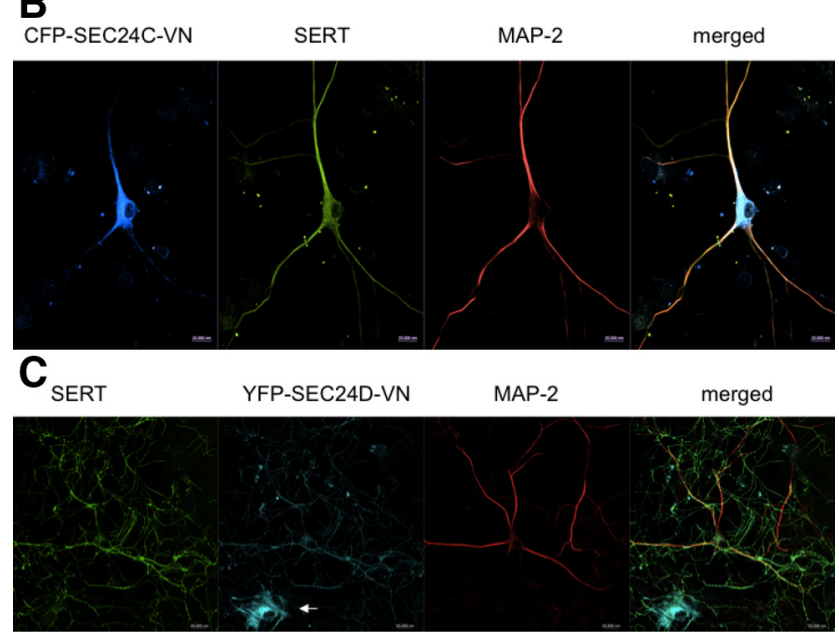

Figure 6. Dominant-negative SEC24C precludes delivery of SERT into the axonal compartment of rat dorsal raphe neurons. Rat dorsal raphe neurons were transfected with plasmids coding for CFP-SEC24C (A), CFP-SEC24C-VN (B), or YFP-SEC24D-VN (C) using Lipofectamine2000. After $48 \mathrm{~h}$, the neurons were fixed and stained for endogenous SERT (H-45) and MAP-2. The immunoreactivity was visualized with Alexa Fluor 568-, Alexa Fluor 488-, or Alexa Fluor 633-conjugated secondary antibodies. In C, the small white arrow highlights a glial cell transfected with YFP-SEC24D-VN only. Images were captured by confocal microscopy. During expression of CFP-SEC24C-VN, SERT was confined to the MAP-2-positive compartment in 18 of 18 examined neurons; in the presence of dominant-negative SEC24D, SERT reached the MAP2 -negative compartment in 14 of 14 examined neurons. Data are from four independent experiments (i.e., 4 individual preparations of rat dorsal raphe neurons that were independently transfected in parallel with plasmids encoding (FP-SEC24C-VN or YFP-SEC24D-VN). Scale bars, $20 \mu \mathrm{m}$.

\section{Discussion}

Neurotransmitter transporters of the SLC6 family share a conserved hydrophobic core that is required for translocation of their cognate substrates but differ substantially in their intracellular $\mathrm{N}$ and $\mathrm{C}$ termini. The large divergence in the intracellular segments is likely to result from differences in sorting, targeting, retention, recycling, and regulatory input, for example, by protein kinases (Kristensen et al., 2011). There are only very few conserved residues in these segments, in particular invariant proline and glycine residues. These presumably reflect constraints imposed by folding. In addition, we previously identified a conserved RI/RL/KL motif in the C terminus of SLC6 family members that was required for ER export of neurotransmitter transporters, because it supported the recruitment of the COPII components SEC24C and SEC24D (Farhan et al., 2007; Sucic et al., 2011). This previous work also showed that GAT-1 required SEC24D to reach the presynaptic specialization of hippocampal neurons (Reiterer et al., 2008). Here we examined SEC24Cdependent targeting of SERT in neurons isolated from the midbrain raphe nuclei, i.e., the very neurons that endogenously express the transporter and that are the predominant source of 5-HT within the brain. We used three complementary ap-
A

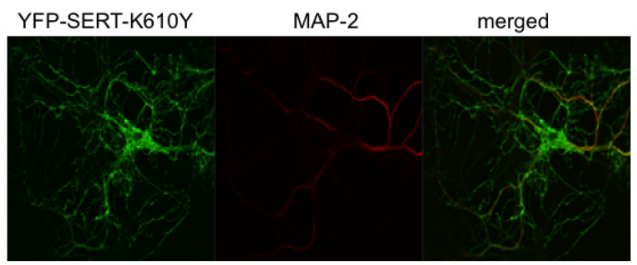

B

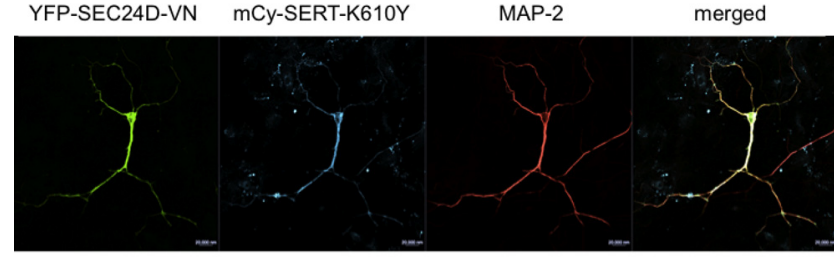

C

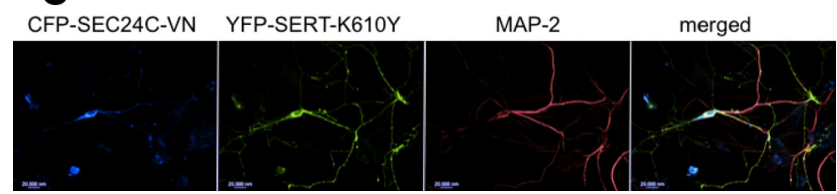

Figure 7. Axonal targeting of YFP-tagged SERT-K ${ }^{610} Y$, an SEC24D client. Rat dorsal raphe neurons were transfected with plasmids encoding YFP-SERT-K ${ }^{610} Y(\boldsymbol{A})$, YFP-SEC24D-VN and mCherry-SERT-K ${ }^{610} Y$ (mCy-SERT-K610Y; plasmid ratio of 5:1; $\boldsymbol{B}$ ), or CFP-SEC24C-VN and YFP-SERT-K $K^{610} Y$ (plasmid ratio of 5:1; C) using Lipofectamine2000. After $48 \mathrm{~h}$, the neurons were fixed and stained for MAP-2. MAP-2 staining was detected using Alexa Fluor 568- or Alexa Fluor 633-conjugated secondary antibodies. Images were captured by confocal microscopy. YFP-tagged SERT-K ${ }^{610} \mathrm{Y}$ reached the MAP-2-negative compartment in 14 of 14 examined and in 15 of 15 examined neurons in the absence and presence of CFP-SEC24C-VN. During coexpression of dominant-negative SEC24D, mCherry-tagged SERT-K ${ }^{610} \mathrm{Y}$ was confined to the MAP-2-positive compartment in 16 of 20 neurons. Data are from four independent experiments (i.e., 4 individual preparations of rat dorsal raphe neurons that were independently transfected in parallel with a plasmid coding for a tagged version of SERT $-K^{610} \mathrm{Y}$ and plasmids encoding (FP-SEC24C-VN or YFP-SEC24D-VN). Scale bars, $20 \mu \mathrm{m}$.

proaches to confirm that axonal delivery of SERT was contingent on SEC24-dependent ER export. (1) Heterologously expressed YFP-tagged SERT faithfully reproduced the localization of endogenous SERT and became enriched in the thin axonal arborizations that are elaborated by the raphe neurons. In contrast, heterologously expressed SERT- ${ }^{607} \mathrm{RI}^{608}-\mathrm{AA}$ was confined to the MAP-2-positive somatodendritic compartment. This mutant fails to recruit SEC24C but eventually reaches the cell surface (Sucic et al., 2011). (2) A dominant-negative version of SEC24C, but not of SEC24D, redirected both endogenous SERT and heterologously expressed YFP-tagged SERT to the somatodendritic compartment. This observation ruled out that the mistargeting of SERT $-{ }^{607} \mathrm{RI}^{608}-\mathrm{AA}$ resulted from its folding deficit (El-Kasaby et al., 2010). (3) SERT-K ${ }^{610} \mathrm{Y}$, a variant engineered to become an SEC24D client, does not incur any folding problem and is functionally indistinguishable from wild-type SERT (Sucic et al., 2013). SERT-K ${ }^{610} Y$ was faithfully enriched in axons but was redirected to the somatodendritic compartment during coexpression of a dominant-negative version of SEC24D. In contrast, dominant-negative SEC24C did not interfere with the enrichment of SERT $-\mathrm{K}^{610} \mathrm{Y}$ in the axonal arborizations. Accordingly, this switch argues against a nonspecific, general disruption of axonal delivery by dominant-negative SEC24C. This is further supported by the observation that neither dominant-negative SEC24C nor dominant-negative SEC24D substantially interfered with axonal delivery of VMAT2. Neurotransmission in the brain has two principle components: (1) wired transmission is mainly accomplished by glutamatergic and GABAergic projection neu- 

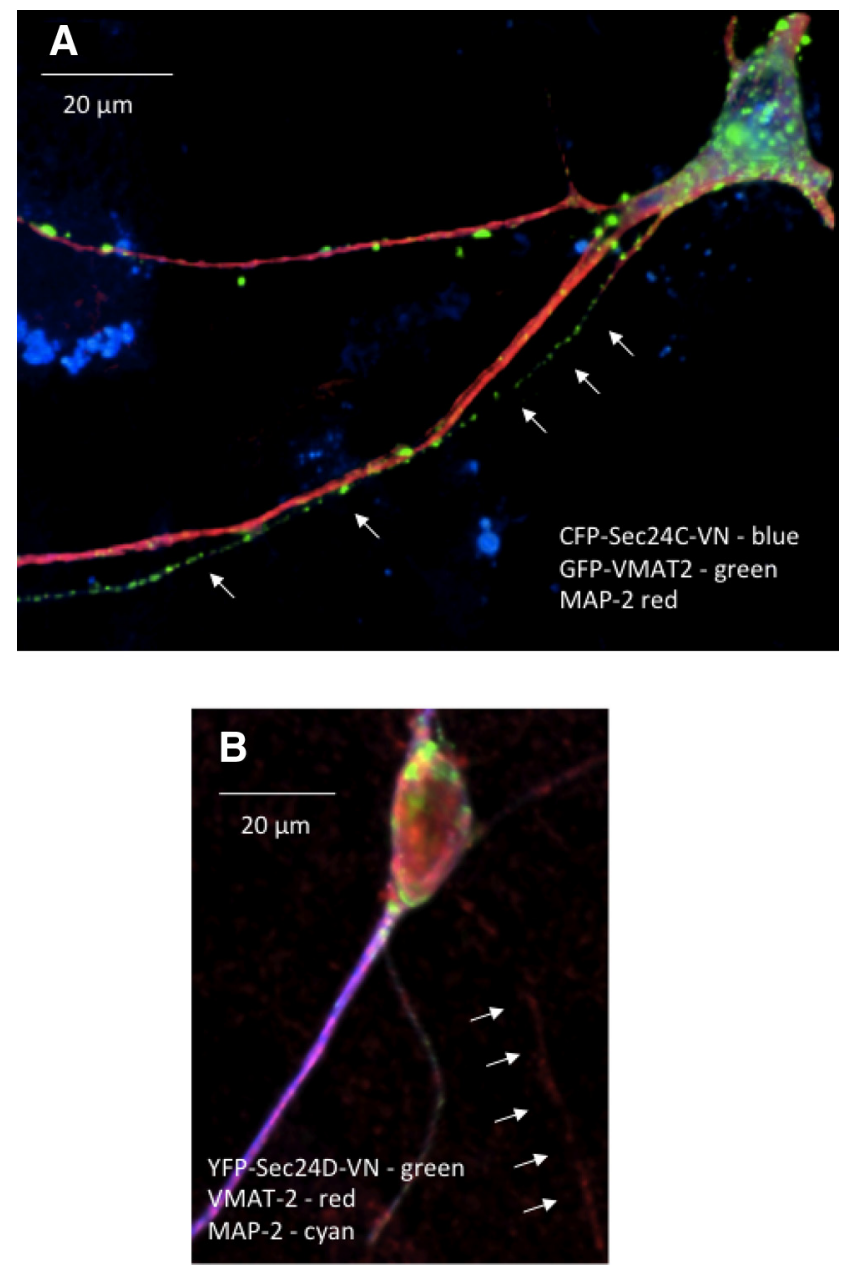

Figure 8. Delivery of GFP-tagged $(\boldsymbol{A})$ and endogenous VMAT2 $(\boldsymbol{B})$ to the MAP-2-negative compartment in the presence of dominant-negative SEC24C $(\boldsymbol{A})$ or dominant-negative SEC24D (B). Rat dorsal raphe neurons were transfected with plasmids encoding GFP-VMAT2 and CFPSEC24C-VN (1:5 ratio; $\boldsymbol{A})$ or YFP-SEC24D-VN $(\boldsymbol{B})$ using Lipofectamine2000. After $48 \mathrm{~h}$, the neurons were fixed and stained for MAP-2 $(\boldsymbol{A}, \boldsymbol{B})$ and VMAT2 $(\boldsymbol{B})$. Alexa Fluor 568-conjugated (A) or Alexa Fluor 405-conjugated (B) secondary antibodies were used to visualize MAP-2; VMAT2 was detected with an Alexa Fluor 568-conjugated secondary antibody $(\boldsymbol{B})$. A $3 \mathrm{D}$ reconstruction was generated from z stacks using NIH Image J to visualize VMAT2 containing vesicles: VMAT2 was unequivocally assigned to the MAP-2-negative compartment in 3 of 8 and 2 of 8 examined neurons coexpressing SEC24C and SEC24D, respectively. Small arrows point to VMAT2 fluorescence present in MAP-2-negative axonal extensions. Data are from three independent experiments (i.e., 3 individual preparations of rat dorsal raphe neurons that were independently transfected in parallel with a plasmid encoding (FP-SEC24C-VN or YFP-SEC24D-VN). Scale bars, $20 \mu \mathrm{m}$.

rons; and (2) monoaminergic neurons produce elaborate axonal trees that make multiple synaptic contacts and thereby support volume transmission (Fuxe et al., 2010). Based on the current observations with SERT and our previous findings with GAT-1 (Reiterer et al., 2008), we therefore conclude that SEC24dependent ER export is a prerequisite for routing SLC6 neurotransmitter transporters to the axonal compartment. This is true regardless of whether the neurotransmitter transporter is part of the machinery required to predominantly support wired transmission (GAT-1) or volume transmission (SERT).

In polarized mammalian cells (i.e., epithelial cells and neurons), cargo, which undergoes anterograde trafficking, must at some point be subject to a sorting decision to reach its final destination. Basolateral and apical membranes of polarized epithelial cells are considered to be equivalent to the somatodendritic and axonal compartment of neurons, respectively. Although this is an oversimplification, it has allowed for deducing common general mechanisms. Cargo segregation can be visualized in the trans-Golgi network (Keller et al., 2001). This suggests that sorting decisions are being made at this level. Our observations indicate that the destination of SERT is already specified at the level of the ER. This apparent discrepancy can be rationalized as follows. Axonal sorting is, at least in part, contingent on the formation of microdomains composed of proteins and lipids, in which cholesterol and glycosphingolipids are enriched and are referred to as (lipid) rafts (Ledesma et al., 1998; Jacob and Naim, 2001). Fullfledged rafts are thought to assemble in the Golgi, because this is the site in which sphingolipid synthesis is accomplished. However, the ceramide precursor is generated in the ER, which is also the site of cholesterol synthesis (Hoetzl et al., 2007). In fact, assembly of raft-like detergent-resistant microdomains has been observed in the ER, and this was required for ER export of the glycosylphosphatidylinositol-anchored cargo proteins CD59 and folate receptor- $\alpha$ (Bonnon et al., 2010). Importantly, export of CD59 and the folate receptor- $\alpha$ was dependent on SEC24C and SEC24D (but not on SEC24A and SEC24B). Based on these observations, Bonnon et al. (2010) put forth the hypothesis that SEC24C and SEC24D preferentially export raft-associated cargo proteins and thus participate in sorting decisions. Our findings are in line with this model, because SEC24D substituted for SEC24C and supported axonal targeting of SERT provided that a point mutation was introduced in the $\mathrm{C}$ terminus of SERT (i.e., $\mathrm{K}^{610}$ to $\mathrm{Y}$ ) to convert it to an SEC24D client. Lipid rafts emanating from the ER are likely to be reshaped in the Golgi cisternae (Hoetzl et al., 2007) and thus undergo another round of sorting in the trans-Golgi network (Keller et al., 2001). It is also worth noting that SERT partitions into lipid microdomains (Magnani et al., 2004), requires cholesterol for activity (Scanlon et al., 2001), and does not fold correctly in its absence (Tate et al., 2003).

Our approach has two limitations. (1) Cultures prepared from the dorsal raphe nuclei are enriched in serotonergic neurons, but they also contain other neurons. In fact, TPH2-positive neurons represent $70 \%$ of the total neuronal population. Thus, the heterologously expressed variants of SERT were not necessarily expressed in serotonergic neurons. However, it is worth pointing out that overexpression of the dominant-negative version of SEC24C (but not of SEC24D) redirected endogenously expressed SERT to the somatodendritic compartment (Fig. 6B, C). At the very least, this finding confirms that cultured serotonergic raphe neurons require SEC24C to deliver SERT to the axonal compartment. (2) Another limitation of our approach is the fact that we used neurons from developing brain. Hence, we cannot formally rule out that the targeting machinery operates in a different manner in the brain of an adult organism. Our findings point to a strategy for addressing the distribution in vivo: if the endogenous SERT gene is replaced with a version encoding SERT- ${ }^{607} \mathrm{RI}^{608}$ $\mathrm{AA}$, the resulting mutated protein is predicted to remain confined to the somatodendritic compartment and thus to be absent in the serotoninergic projection areas within the brain of an adult organism. Neurotransmitter transporters of the SLC6 family can also be found in the somatodentritic compartment of the plasma membrane, although it is less clear what their physiological role may be at this site (Kristensen et al., 2011; Colgan et al., 2012). We also visualized SERT on the soma of raphe neurons. At the very least, the strategy outlined above allows for testing their relevance, if the mutant SERT is indeed confined to the somatodendritic compartment. The neurotoxic actions of MDMA and its congeners are, for instance, essentially limited to the axons of 
serotonergic neurons (O'Hearn et al., 1988; for review, see Steinkellner et al., 2011). However, MDMA treatment affects serotonergic markers (e.g., VMAT2 and SERT) in a differential manner (Biezonski and Meyer, 2010). It will be of interest to determine whether a somatically confined SERT variant modifies the toxicity of MDMA. Similarly, the antidepressant action of SSRIs are thought to result from their action on presynaptic retrieval of 5-HT. This predicts that they should be devoid of any action if SERT is confined to the somatodendritic compartment. These issues are currently being addressed.

\section{References}

Anitei M, Hoflack B (2012) Bridging membrane and cytoskeleton dynamics in the secretory and endocytic pathways. Nat Cell Biol 14:11-19. CrossRef Medline

Biezonski DK, Meyer JS (2010) Effects of 3,4-methylenedioxymethamphetamine (MDMA) on serotonin transporter and vesicular monoamine transporter 2 protein and gene expression in rats: implications for MDMA neurotoxicity. J Neurochem 112:951-962. CrossRef Medline

Bonnon C, Wendeler MW, Paccaud JP, Hauri HP (2010) Selective export of human GPI-anchored proteins from the endoplasmic reticulum. J Cell Sci 123:1705-1715. CrossRef Medline

Colgan LA, Cavolo SL, Commons KG, Levitan ES (2012) Action potentialindependent and pharmacologically unique vesicular serotonin release from dendrites. J Neurosci 32:15737-15746. CrossRef Medline

El-Kasaby A, Just H, Malle E, Stolt-Bergner PC, Sitte HH, Freissmuth M, Kudlacek O (2010) Mutations in the carboxyl-terminal SEC24 binding motif of the serotonin transporter impair folding of the transporter. J Biol Chem 285:39201-39210. CrossRef Medline

Eriksen J, Rasmussen SG, Rasmussen TN, Vaegter CB, Cha JH, Zou MF, Newman AH, Gether U (2009) Visualization of dopamine transporter trafficking in live neurons by use of fluorescent cocaine analogs. J Neurosci 29:6794-6808. CrossRef Medline

Farhan H, Korkhov VM, Paulitschke V, Dorostkar MM, Scholze P, Kudlacek O, Freissmuth M, Sitte HH (2004) Two discontinuous segments in the carboxy terminus are required for membrane targeting of the rat GABA transporter-1 (GAT1). J Biol Chem 279:28553-28563. CrossRef Medline

Farhan H, Reiterer V, Korkhov VM, Schmid JA, Freissmuth M, Sitte HH (2007) Concentrative export from the endoplasmic reticulum of the GABA-transporter 1 requires binding to SEC24D. J Biol Chem 282:76797689. CrossRef Medline

Farhan H, Reiterer V, Kriz A, Hauri HP, Pavelka M, Sitte HH, Freissmuth M (2008) Signal dependent export of the GABA transporter-1 from the intermediate compartment is specified by a carboxyl terminal motif. J Cell Sci 121:753-761. CrossRef Medline

Fuxe K, Dahlström AB, Jonsson G, Marcellino D, Guescini M, Dam M, Manger P, Agnati L (2010) The discovery of central monoamine neurons gave volume transmission to the wired brain. Prog Neurobiol 90:82-100. CrossRef Medline

Gillon AD, Latham CF, Miller EA (2012) Vesicle-mediated ER export of proteins and lipids. Biochim Biophys Acta 1821:1040-1049. CrossRef Medline

Hoetzl S, Sprong H, van Meer G (2007) The way we view cellular (glyco)sphingolipids. J Neurochem 103:3-13. CrossRef Medline

Jacob R, Naim HY (2001) Apical membrane proteins are transported in distinct vesicular carriers. Curr Biol 11:1444-1450. CrossRef Medline

Keller P, Toomre D, Díaz E, White J, Simons K (2001) Multicolour imaging of post-Golgi sorting and trafficking in live cells. Nat Cell Biol 3:140-149. CrossRef Medline

Kristensen AS, Andersen J, Jørgensen TN, Sørensen L, Eriksen J, Loland CJ, Strømgaard K, Gether U (2011) SLC6 neurotransmitter transporters: structure, function, and regulation. Pharmacol Rev 63:585-640. CrossRef Medline

Kroeze Y, Zhou H, Homberg JR (2012) The genetics of selective serotonin reuptake inhibitors. Pharmacol Ther 136:375-400. CrossRef Medline
Ledesma MD, Simons K, Dotti CG (1998) Neuronal polarity: essential role of protein-lipid complexes in axonal sorting. Proc Natl Acad Sci U S A 95:3966-3971. CrossRef Medline

Magnani F, Tate CG, Wynne S, Williams C, Haase J (2004) Partitioning of the serotonin transporter into lipid microdomains modulates transport of serotonin. J Biol Chem 279:38770-38778. CrossRef Medline

Minelli A, Brecha NC, Karschin C, DeBiasi S, Conti F (1995) GAT-1, a high-affinity GABA plasma membrane transporter, is localized to neurons and astroglia in the cerebral cortex. J Neurosci 15:7734-7746. Medline

Miner LH, Schroeter S, Blakely RD, Sesack SR (2000) Ultrastructural localization of the serotonin transporter in superficial and deep layers of the rat prelimbic prefrontal cortex and its spatial relationship to dopamine terminals. J Comp Neurol 427:220-234. CrossRef Medline

O’Hearn E, Battaglia G, De Souza EB, Kuhar MJ, Molliver ME (1988) Methylenedioxyamphetamine (MDA) and methylenedioxymethamphetamine (MDMA) cause selective ablation of serotonergic axon terminals in forebrain: immunocytochemical evidence for neurotoxicity. J Neurosci 8:2788-2803. Medline

Radian R, Ottersen OP, Storm-Mathisen J, Castel M, Kanner BI (1990) Immunocytochemical localization of the GABA transporter in rat brain. J Neurosci 10:1319-1330. Medline

Rayport S, Sulzer D, Shi WX, Sawasdikosol S, Monaco J, Batson D, Rajendran G (1992) Identified postnatal mesolimbic dopamine neurons in culture: morphology and electrophysiology. J Neurosci 12:4264-4280. Medline

Reiterer V, Maier S, Sitte HH, Kriz A, Rüegg MA, Hauri HP, Freissmuth M, Farhan H (2008) Sec24- and ARFGAP1-dependent trafficking of GABA transporter-1 is a prerequisite for correct axonal targeting. J Neurosci 28:12453-124564. CrossRef Medline

Scanlon SM, Williams DC, Schloss P (2001) Membrane cholesterol modulates serotonin transporter activity. Biochemistry 40:10507-10513. CrossRef Medline

Steinkellner T, Freissmuth M, Sitte HH, Montgomery T (2011) The ugly side of amphetamines: short- and long-term toxicity of 3,4-methylenedioxymethamphetamine (MDMA, "Ecstasy"), methamphetamine and D-amphetamine. Biol Chem 392:103-115. CrossRef Medline

Sucic S, El-Kasaby A, Kudlacek O, Sarker S, Sitte HH, Marin P, Freissmuth M (2011) The serotonin transporter is an exclusive client of the coat protein complex II (COPII) component SEC24C. J Biol Chem 286:16482-16490. CrossRef Medline

Sucic S, Koban F, El-Kasaby A, Kudlacek O, Stockner T, Sitte HH, Freissmuth M (2013) Switching the clientele: a lysine residing in the C-terminus of the serotonin transporter specifies its preference for the COPII component SEC24C. J Biol Chem 288:5330-5341. CrossRef Medline

Tao-Cheng JH, Zhou FC (1999) Differential polarization of serotonin transporters in axons versus soma-dendrites: an immunogold electron microscopy study. Neuroscience 94:821-830. CrossRef Medline

Tate CG, Haase J, Baker C, Boorsma M, Magnani F, Vallis Y, Williams DC (2003) Comparison of seven different heterologous protein expression systems for the production of the serotonin transporter. Biochim Biophys Acta 1610:141-153. CrossRef Medline

Walther DJ, Bader M (2003) A unique central tryptophan hydroxylase isoform. Biochem Pharmacol 66:1673-1680. CrossRef Medline

Yasufuku-Takano J, Nakajima S, Nakajima Y (2008) Morphological and physiological properties of serotonergic neurons in dissociated cultures from the postnatal rat dorsal raphe nucleus. J Neurosci Methods 167:258 267. CrossRef Medline

Zanetti G, Pahuja KB, Studer S, Shim S, Schekman R (2012) COPII and the regulation of protein sorting in mammals. Nat Cell Biol 14:20-28. CrossRef Medline

Zhou FC, Tao-Cheng JH, Segu L, Patel T, Wang Y (1998) Serotonin transporters are located on the axons beyond the synaptic junctions: anatomical and functional evidence. Brain Res 805:241-254. CrossRef Medline

Zhuang X, Masson J, Gingrich JA, Rayport S, Hen R (2005) Targeted gene expression in dopamine and serotonin neurons of the mouse brain. J Neurosci Methods 143:27-32. CrossRef Medline 